Western University

Scholarship@Western

Communication Sciences and Disorders

Publications

Communication Sciences and Disorders School

$7-1-2018$

\title{
Immature auditory evoked potentials in children with moderate-severe developmental language disorder
}

\author{
Elaine Y.L. Kwok \\ The University of Western Ontario \\ Marc F. Joanisse \\ The University of Western Ontario \\ Lisa M.D. Archibald \\ The University of Western Ontario \\ Janis Oram Cardy \\ The University of Western Ontario
}

Follow this and additional works at: https://ir.lib.uwo.ca/scsdpub

Citation of this paper:

Kwok, Elaine Y.L.; Joanisse, Marc F.; Archibald, Lisa M.D.; and Cardy, Janis Oram, "Immature auditory evoked potentials in children with moderate-severe developmental language disorder" (2018).

Communication Sciences and Disorders Publications. 18.

https://ir.lib.uwo.ca/scsdpub/18 
Western University

Scholarship@Western

Health and Rehabilitation Sciences Publications

Health and Rehabilitation Sciences Program

7-13-2018

\section{Immature Auditory Evoked Potentials in Children With Moderate-Severe Developmental Language Disorder.}

Elaine Yuen Ling Kwok

Health and Rehabilitation Science, Western University, ekwok5@uwo.ca

Marc F Joanisse

Lisa Archibald

Western University, larchiba@uwo.ca

Janis Oram Cardy

Follow this and additional works at: https://ir.lib.uwo.ca/hrspub

Part of the Cognitive Neuroscience Commons, Developmental Neuroscience Commons, Rehabilitation and Therapy Commons, and the Speech Pathology and Audiology Commons

Citation of this paper:

Kwok, E. Y., Joanisse, M. F., Archibald, L. M., \& Cardy, J. O. (2018). Immature Auditory Evoked Potentials in Children With Moderate-Severe Developmental Language Disorder. Journal of Speech, Language, and Hearing Research, 61(7), 1718-1730. 
Immature auditory evoked potentials in children with moderate-severe developmental language disorder

Elaine Y. L. Kwok, Marc F. Joanisse, Lisa M. D. Archibald, \& Janis Oram Cardy

\section{Author Note}

Elaine Y. L. Kwok, Graduate Program in Health and Rehabilitation Sciences and School of Communication Sciences and Disorders, The University of Western Ontario; Marc F. Joanisse, Department of Psychology and Brain and Mind Institute, The University of Western Ontario; Lisa M. D. Archibald, School of Communication Sciences and Disorders and Department of Psychology, The University of Western Ontario; Janis Oram Cardy, School of Communication Sciences and Disorders, The University of Western Ontario.

This research was supported by the Natural Sciences and Engineering Research Council of Canada Discovery Grant 418406-2012 to JOC and 371201-2009 to LMDA, and a Scottish Rite Charitable Foundation of Canada Research Grant to JOC.

Correspondence concerning this article should be addressed to: Janis Oram Cardy, School of Communication Sciences and Disorders, The University of Western Ontario, Elborn College, London, Ontario, Canada, N6G 1H1. Email: janis.cardy@uwo.ca 


\begin{abstract}
Purpose: Immature auditory processing has been proposed to underlie language impairments in children with developmental language disorder (DLD; also known as specific language impairment). Using newly available normative auditory evoked potential (AEP) waveforms, we estimated AEP maturity in individual children with DLD and explored whether this maturational index was related to their language abilities.
\end{abstract}

Method: AEPs were elicited by 225 trials of a $490 \mathrm{~Hz}$ pure tone. Using ICC and our previously established normative AEP waveforms of 7- to 10-year-old children with typical development, we estimated the age-equivalent of the AEPs (AEP-age) from 21 children with DLD. The relation between AEP maturity and language was explored through regression analysis.

Results: AEP-age predicted $31 \%$ of the variance in the language abilities of children with DLD. The AEP-age of children with mild DLD was similar to their chronological age, whereas children with moderate-severe DLD showed, on average, a 1.3 year delay in their neural responses. AEP-age predicted receptive, but not expressive, language performance.

Conclusions: Maturation in auditory neural responses is a significant predictor of language ability, particularly in children with moderate-severe DLD.

Keywords: language impairment, developmental language disorder, DLD, auditory processing, event-related potentials 
Immature auditory evoked potentials in children with moderate-severe developmental language disorder

A number of studies examining children with developmental language disorder (DLD; also known as specific language impairment; Bishop, Snowling, Thompson, Greenhalgh, \& The CATALISE-2 Consortium, 2017), have suggested that central auditory function contributes to language ability. That is, children with language impairments who have normal peripheral hearing have nonetheless demonstrated atypical responses to various auditory stimuli in both psycho-behavioural (see Tallal, 2000 for review) and neurophysiological paradigms (e.g., Archibald \& Joanisse, 2012; Bishop, Hardiman, Uwer, \& von Suchodoletz, 2007; Bishop \& McArthur, 2004, 2005; Choudhury \& Benasich, 2011; Lincoln, Courchesne, Harms, \& Allen, 1995; McArthur \& Bishop, 2004a, 2005; Neville, Coffey, Holcomb, \& Tallal, 1993; Oram Cardy, Flagg, Roberts, Brian, \& Roberts, 2005; Oram Cardy, Flagg, Roberts, \& Roberts, 2008). These findings have led some to suggest that difficulties in processing acoustic elements within speech are key contributors to DLD. Under this view, an underlying auditory processing impairment is proposed to limit the quality of linguistic input from an auditory perceptual standpoint, thereby interfering with the development of the phonological system, and, in turn, spoken and written language (e.g., Joanisse, 2004; Leonard, 2014; Tallal, 2000).

The theory of immature auditory processing (McArthur \& Bishop, 2004b) hypothesizes that children with DLD have a general maturational delay in their auditory development, which contributes to both their impaired performance on auditory judgment tasks (such as rapid temporal perception tasks) and their delayed language development. A handful of studies found evidence supporting this hypothesis. For example, Wright and Reid (2002) found that 12-year- 
old children with DLD had similar thresholds for simultaneous, backward, and forward masking to 8-year-old controls, which was significantly different from the performances of age-matched controls. Corriveau, Pasquini, and Goswami (2007) found that children with DLD ( $M=10$ years) performed significantly worse than age-matched controls but no different from younger children matched on language age ( $M=7$ years) on a number of auditory tasks targeting the processing of amplitude envelope onset rise time and duration.

Beyond methodologies that require behavioral responses (e.g., through button press), an alternate approach to examining auditory maturation involves using electroencephalography (EEG), which records the obligatory neural responses that are evoked by auditory stimuli. These neural responses, termed auditory evoked potentials (AEPs), generate electrical voltages that are recorded on the scalp and averaged across many presentations of the same auditory stimulus. Since AEPs can be obtained through passive paradigms (i.e., where children do not need to actively attend or respond to the stimuli), they have the potential to be used in populations of younger or clinically more complex children. Amongst the known components of cortical AEPs, some are thought to be related to the detection of auditory stimuli, including P1, a positive deflection occurring approximately $80-110 \mathrm{~ms}$ post-stimulus in children; N1, a negative deflection $\sim 120$-150ms post-stimulus; P2, a positive deflection $\sim 140-160$ ms post stimulus; and N2, a negative deflection 220-250ms post-stimulus (Ponton, Eggermont, Kwong, \& Don, 2000). Of these four components, $\mathrm{P} 1$ and $\mathrm{N} 2$ have been shown to be the most predominant AEP components in children with typical development aged 8 to 11 years (Sussman, Steinschneider, Gumenyuk, Grushko, \& Lawson, 2008) and to undergo significant maturational changes in amplitude and latency during the school-age years (Ponton et al., 2000). In contrast, components N1 and P2 only become identifiable in children aged 9 years or older (Ponton et al., 2000). 
These age-related development and emergence of AEP components reflects anatomical maturation in the auditory cortex which may underlie the improvements in auditory perception during childhood (Eggermont \& Ponton, 2003; Moore 2002). Indeed, P1, N1, P2, N2 and other AEP components such as mismatch negativity (MMN), have been used to index specific auditory processes in children with DLD, including temporal and frequency discrimination (e.g., Bishop \& McArthur, 2004; Ceponiene, Cummings, Wulfeck, Ballantyne, \& Townsend, 2009; Duffy, Eksioglu, \& Rotenberg, 2013; also see reviews by Bishop, 2007; Uwer et al., 2002). AEP components that show significant changes during the developmental time period of interest and that relate to simple detection (rather than more fine-grained perceptual discrimination of specific features) can be useful indices of overall auditory processing maturity. Investigation into these neural responses allows for a way to examine the immature auditory processing hypothesis of language impairment without the confounding factors of behavioral paradigms.

The current literature found inconsistent support for difference in AEPs between children with typical development and children with DLD (see Bishop et al., 2007; McArthur \& Bishop, 2004b; McArthur, Atkinson, \& Ellis, 2009 for reviews). For example, Ors et al. (2002) found no difference in N1 and P2 components between a group of children with DLD and those with typical development. Other studies, however, have demonstrated a difference in AEPs in these groups (e.g., Tonnquist-Uhlén, 1996; Wible, Nicol, \& Krause, 2005), and some have additionally demonstrated a relation between aspects of AEPs and language ability (e.g., McArthur \& Bishop, 2004b, 2005; McArthur et al., 2009; Oram Cardy et al., 2008). For example, using a non-speech pure tone as the auditory stimulus and magnetoencephalography (MEG) to record cortical responses, Oram Cardy et al. (2008) found that delay in the latency of the M50 peak (the magnetic equivalent of P1) was associated with language impairment. The latency of M50 alone 
predicted $36 \%$ of the variance in the language performance of children and adolescents.

Heterogeneity in the DLD population is one possible explanation for inconsistent findings across studies. Neville et al. (1993) proposed that only a subset of children with DLD have an underlying auditory processing immaturity. Given that different AEP components are more or less prominent at different periods of child development and can relate to different stages of stimulus processing, analysis of different AEP components and of children at different ages across studies may have also contributed to discrepant findings in the literature. Furthermore, majority of current studies have compared aspects of AEPs (i.e., peak amplitude, timing, or topography) between children with DLD to same-age children with typical development (but not to older or younger control groups), it remains unclear whether the AEP found children with DLD were immature as predicted by the immature auditory processing hypothesis.

McArthur and Bishop (2004b) proposed a novel way to analyse AEPs through the use of intraclass correlation (ICC) calculation, which measures the global resemblance of two waveforms. Compared to traditional analysis of individual components, ICC allows researchers to specify a time window to be compared between two waveforms while avoiding the need to isolate particular AEP components. This particular characteristic of ICC allows for comparison across children of different ages ( since some AEP components such as N1 and P2 may not be identifiable until past 9 years of age), and comparison of morphologically different waveforms from populations with neurodevelopmental disorders (e.g., significantly flatter AEP waveforms were found in children with DLD and reading disability compared to same-age typical controls by McArthur et al., 2009). Using the ICC approach, Bishop, Anderson, Reid, and Fox (2011) demonstrated that it is possible to assign age-equivalents to the AEP responses of individual children with typical development aged 7, 9, and 11 years. The estimated AEP age-equivalents 
were found to be reliable predictors of chronological age, thus capturing maturation.

Additionally, when compared to traditional analytical methodologies such as time-frequency or independent component analysis, ICC analysis alone allowed for generation of an AEP maturity estimate at the level of the individual child (Bishop, Anderson, Reid, \& Fox, 2011). The sensitivity and reliability of AEP age-equivalent estimation in individual children via ICC was replicated in our study of children with typical development aged 7, 8, 9, and 10 years (Kwok et al., 2018).

Application of the ICC approach in the DLD literature has been limited, albeit promising, to date. McArthur and Bishop (2004) found that AEP waveforms of adolescents with DLD in response to a simple tone differed from same-age peers with typical development. This finding was replicated in school-age children with DLD (5-10 years old) using speech stimuli (Bishop et al., 2007). Further investigation revealed that some individuals with DLD had AEP waveforms that were more similar to younger peers with typical development (Bishop \& McArthur, 2005). Lastly, McArthur, Atkinson and Ellis (2009) found that children with DLD had AEP-waveforms that differed from controls at electrode C3. To date, no study has attempted to estimate AEP ageequivalent in individual children with DLD, as has been completed by Bishop et al. (2011) and Kwok et al. (2018) in children with typical development. Doing so would provide an opportunity to evaluate the immature auditory processing account of DLD in a way that avoids some of the confounds associated with attempting to isolate individual AEP components, which may have contributed to conflicting findings in past neurophysiological studies. Further, generating an estimate of auditory cortical maturity for individual children with DLD would allow for more nuanced exploration of its relation to language ability in this population than the broad DLD versus typical development group comparisons conducted to date. 
In the present study, we examined the immature auditory processing account of DLD by measuring auditory cortical responses to a single pure tone in a group of 7-10-year-old children with DLD. The AEPs of children with DLD were compared to our previously reported normative waveforms of children with typical development (Kwok et al., 2018) using ICC, and an estimate of each child's auditory cortical maturity (i.e., a "brain age equivalent") was generated. Using these AEP maturity estimates, we then examined four research questions: (a) Do children with DLD differ from children with typical development in their AEP maturity overall? (b) Can we predict language ability in children with DLD using AEP maturity? (c) Are AEP waveforms more immature or only immature in children with moderate-severe DLD compared to children with mild DLD? and (d) Is AEP maturity in children with DLD related to receptive language ability, expressive language ability, or both?

In line with previous evidence supporting models of delay in language, perceptual, and motor development in children with DLD (Bishop \& Edmundson, 1987; Rice, Wexler, \& Hershberger, 1998; Rubin, Kantor, \& Macnab, 1990; Smith-Lock, 1993), we predicted that AEPs in children with DLD would be delayed relative to same-aged peers. In line with the immature auditory processing account of DLD, we predicted that waveform maturation would be a significant predictor of language ability and that children with DLD who had moderate-severe language impairments would show more immature AEPs relative to those with mild language impairments. We also considered the possibility that AEP immaturity might only be evident in children with more substantial degrees of language impairment, that is, using a more conservative cut-off of 1.25 SD below the mean (Reilly et al., 2014). In line with prior evidence linking cortical auditory processing specifically to receptive language ability (Bishop et al., 
2007; Oram Cardy et al., 2008), we hypothesized that AEP maturity would predict receptive but not expressive language ability.

\section{Participants}

\section{Methods}

Children with DLD. Twenty-one 7- to 10-year-old children with DLD participated. All children were primary English speakers with no neurological, hearing, or visual impairment by parental report. All participants completed the Wechsler Abbreviated Intelligence Scale (WASI; Wechler, 1999) and Clinical Evaluation of Language Fundamentals-4 (CELF-4; Semel, Wiig, \& Secord, 2003). To be included in the study, participants must have received a CELF-4 Core Language below 85 (i.e., more than $1 S D$ below the mean) and WASI Performance IQ (PIQ) standard score at or above 85 (i.e., no more than $1 S D$ below the mean). WASI PIQ rather than Full Scale IQ was used to estimate intelligence in this group of children in order to avoid confounding language impairment with IQ estimation (DeThorne \& Schaefer, 2004).

Nineteen of the children with DLD were recruited from, and completed standardized testing as a part of a large-scale, epidemiological investigation of the language, reading, and arithmetic skills of 4- to 10-year-old children in a local school board (Archibald, Oram Cardy, Joanisse, \& Ansari, 2013). We contacted families of children from this study who scored $1 S D$ below the mean on the CELF-4 Core Language score and who consented to be contacted regarding future research opportunities. The remaining three participants were recruited from personal contacts, and completed the standardized tests at the university lab. To rule out the possibility of autism spectrum disorder, all participants scored below the cut-off on the Social Communication Questionnaire Lifetime Form (Rutter \& Bailey, 2003), a caregiver-completed screening tool. 
For analyses related to our third research question, we divided our sample in two severity subgroups using the $C E L F-4$ Core Language score. The inclusion criterion for our overall DLD group was chosen according to the suggested cut-off on the CELF-4 (i.e., $1 S D$ below mean, equivalent to the $16^{\text {th }}$ percentile). Several groups have argued for a more stringent cut-off of 1.25 SD (roughly the $10^{\text {th }}$ percentile) as a clinical reference for children with DLD (see Reilly et al., 2014). Therefore, we used this cut-off to divide our children with DLD into what we have termed mild DLD ( $n=9$, whose $C E L F-4$ Core Language score fell between the $11-16^{\text {th }}$ percentiles) and moderate-severe DLD ( $n=12$, whose CELF-4 Core Language score fell at or below the $10^{\text {th }}$ percentile).

Children with typical development (TD). The normative sample against which the group with DLD was compared was drawn from our previously reported sample of 67 children with TD ( 7 years, $n=15 ; 8$ years, $n=16$; 9 years, $n=22 ; 10$ years, $n=14$ ). More details about this sample can be found in Kwok et al. (in press). Relevant to the present study, the children with TD met identical inclusion criteria to those described above for the group with DLD, except that they received CELF-4 Core Language scores and WASI Full Scale IQ and Verbal IQ scores at or above 85 .

\section{Procedure}

225 trials of a pure tone $(490 \mathrm{~Hz}, 50 \mathrm{~ms}$ duration, $10 \mathrm{~ms}$ onset/offset amplitude ramps $)$ with interstimulus intervals jittered between 1000-1400ms (in 100ms increments) were presented binaurally via inserted earphones. Stimuli were presented at $50 \mathrm{~dB}$ above each child's individual hearing threshold (i.e., dB SL). Throughout the experiment, children watched a silent movie projected on a computer screen at a comfortable viewing distance, and were not required to pay attention to or engage in any task involving the tones. 


\section{EEG Acquisition and Processing}

Event-related encephalograms were recorded at a $250 \mathrm{~Hz}$ sample rate using a 128 -channel DenseArray system with HydroCel Geodesic Sensor Nets (Electrical Geodesics Inc., Eugene, OR, USA). Online and offline filtering was performed, and trials with high impedance ( $>75 \mathrm{k} \Omega$ ) and ocular artifacts were rejected (i.e. eye blinks and eye movements that created sudden spikes in electrical energy of $50 \mu \mathrm{V}$ or greater were identified and removed using a sliding window of $80 \mathrm{~ms})$. The resulting trials were then averaged together for each participant. On average, the AEP waveform of each child with DLD consist of 107 artifact-free EEG trials. Four normative grand average waveforms were created by averaging the AEP waveforms of sixty-seven children with typical development in each of the 7-, 8-, 9-, and 10-year-old age groups (Kwok et al., 2018). On average, the AEP waveform of each child with typical development consist of 110 artifact-free EEG trials.

\section{Analysis of ICC and AEP-Age}

The AEPs of each child with TD or DLD were individually compared to the four normative grand average waveforms. In the case of children with TD, the waveform of the child under comparison was taken out of the normative grand average for that child's chronological age to avoid artificial inflation in similarity. ICC was used as an indicator of overall resemblance of each child's waveform to the each of the four normative grand averages, which allowed for estimation of AEP age-equivalent. For example, an 8-year-old child's AEP waveform may have ICC values of $0.66,0.85,0.78$, and 0.77 when compared to the normative grand average waveforms of 7-, 8-, 9-, and 10-year-old children, respectively. The comparison to the 8-year-old normative grand average yielded the largest ICC value (0.85) for this child, so the estimated ageequivalent for this child's AEP waveform was determined to be 8 years. 
The equation for ICC calculation used was the same as reported by McArthur and Bishop (2004). To capture the P1 and N2 components in the AEPs, ICC was computed 0-500ms poststimulus onset at the three frontal electrodes (F3, Fz, F4). Frontal electrodes were chosen for analysis for several reasons. First, frontal electrodes were most commonly used across studies on AEPs. Second, past studies of participants around the 7-10 year age range used in our study have demonstrated that AEPs were strongest at frontal electrodes, and developmental changes in P1 and N2 were observed at frontal electrodes (Ceponiene, Rinne, \& Näätänen, 2002; Ponton et al., 2000; Sussman et al., 2008). Furthermore, past studies on DLD populations (McArthur \& Bishop, 2004b, 2005) have found significant age-inappropriate waveforms at the Fz channel. The choice of three frontal electrode sites, that is, including locations on the left (F3) and right (F4) regions of the scalp in addition to the central $\mathrm{Fz}$ channel, also provided a more comprehensive representation of frontal neural activity.

To determine the age-equivalent of the AEP of each child with DLD, the child's AEP for each channel was compared to each of the four normative grand averages, and the one that yielded the maximum ICC was assigned as the child's age-equivalent for that channel. That is, the child was assigned the age in years (i.e., 7, 8, 9, or 10) associated with the normative grand average that their waveform most resembled, a concept similar to assigning an age-equivalent according to the raw score obtained on a standardized test. The resulting three age-equivalents (one from each channel) were averaged together to generate the child's overall AEP age equivalent (AEP-age).

\section{Statistical Analysis of AEP-age}

To address our first research questions, we compared our group of children with DLD to our previously reported sample of children with TD on demographic variables and AEP-age 
using independent samples t-tests. To examine our second research question, we explored the relation between chronological age, AEP-age, and language ability by conducting a hierarchical regression analysis to predict variance in $C E L F-4$ Core Language scores. In step 1 of the hierarchical regression analysis, only chronological age was entered as a predictor of language ability. Both chronological age and AEP-age were included in step 2 in order to explore whether additional variance in language ability could be explained above and beyond the effect of developmental maturation. We elected not to include PIQ as an additional predictor in our regression analysis because: (a) in our previous paper on children with TD (Kwok et al., in press), PIQ was not a significant predictor of AEP-age; (b) in our current dataset, PIQ was not correlated with the age-equivalents at any of the three frontal channels; and (c) our sample of 21 children with DLD did not provide adequate power for a third predictor in our analysis. To address our third research question, we compared our group of children with mild DLD to those with moderate-severe DLD on demographic variables and AEP-age using independent samples ttests.

Lastly, to address our fourth research question, we examined the ability of AEP-age to predict receptive and expressive language abilities. Regression analysis was conducted on all participants with DLD using AEP-age as a predictor of receptive and expressive language scores. We used the CELF-4 Concept and Following Directions subtest as an estimate of receptive language ability and the Recalling Sentences subtest as an estimate of expressive language ability. For receptive language, the Concept and Following Directions subtest was the only subtest completed by all participants. For expressive language, we elected to use Recalling Sentences rather than the Formulated Sentences subtest (the only two expressive language subtests completed by all participants) as this subtest has been shown to be most sensitive in 
differentiating children with language impairments from those without (Conti-Ramsden, Botting, \& Faragher, 2001).

\section{Results}

\section{Preliminary Analysis}

We acknowledge that there may be inherent limitations in assigning age-equivalents to the AEP waveforms of children with DLD through referencing them to the normative waveforms of children with typical development. For one, if the AEP waveforms of children with DLD are deviant from those with typical development, estimating an AEP age-equivalent in this case would be similar to measuring weight with a tape measure. Alternatively, if children with DLD follow the typical AEP maturational pattern, we can still be limited by our available normative waveforms. For example, if an 8-year-old child with DLD has an AEP waveform that lags 2 years in development, the age-equivalent of the AEP waveform of this child should be 6 years. However, limited by our available grand averages of 7-, 8-, 9- and 10-year-old children, the lowest age-equivalent we could assign to this child's waveform would be 7 years old. In this case, the age-equivalent estimation is akin to measuring height with a broken tape measure.

Cautious of these potential confounds, we conducted a preliminary analysis to determine whether our available normative AEP waveforms could fully represent the range of developmental maturation in our group of participants with DLD. In both the aforementioned confounds, one would expect to see low ICC values across all age comparisons due to lack of similarity between AEP responses of children with DLD and any of our available normative waveforms. Naturally, individual variation in AEP waveforms is expected even for children with TD who are similar in age. Such variations will result in a distribution of ICCs when comparing typical children's waveforms to an age-appropriate normative grand average. Therefore, we 
determined the normal range of ICC variation among typical controls for comparison with the distribution of ICCs in children with DLD. To do this, the distribution of maximum ICC (out of the four normative grand average comparisons) of the three selected frontal channels were first Fisher's z-transformed using the equation $\mathrm{z}=0.5^{*}[\ln (1+\mathrm{ICC})-\ln (1-\mathrm{ICC})]$. Fisher's $\mathrm{z}-$ transformation ensures the normal distribution of ICC values. Next, these three Fisher's ztransformed ICCs were averaged and compared between children with DLD and TD using an independent samples t-test.

Our decision to compare maximum ICC was based on two considerations: (a) past research suggested that the ICC resulting from comparisons to age-matched normative waveforms would be lower in the group with DLD, and (b) maximum ICC represented the comparison that yielded the highest resemblance between two AEP waveforms. Hence, analyzing maximum ICC is similar to comparing children with DLD to typical controls who are most closely matched in auditory processing maturity. If we found no difference in the distribution of maximum ICC between the two groups, it would suggest that most of our participants with DLD have AEPs that resemble one of our available normative waveforms. However, if a difference was found, it would suggest that our available normative AEP waveforms are not sufficient for estimating the development of AEPs in our group of participants with DLD. In that case, we would have to expand our recruitment of children with TD to establish the normative waveforms in a broader age range.

Overall, as shown in Figure 1, the distribution of maximum ICC values between participants with DLD and TD greatly overlaps. No differences were found between the Fisher's z-transformed maximum ICCs obtained for the group with DLD $(M=1.16, S D=.33)$ and typical controls $(M=1.03, S D=.31), t(86)=-1.55, p=.125$. This suggests that, despite having only 
four normative waveforms based on four age groups, this was sufficient for capturing the maturity of AEPs in our sample of children with DLD.

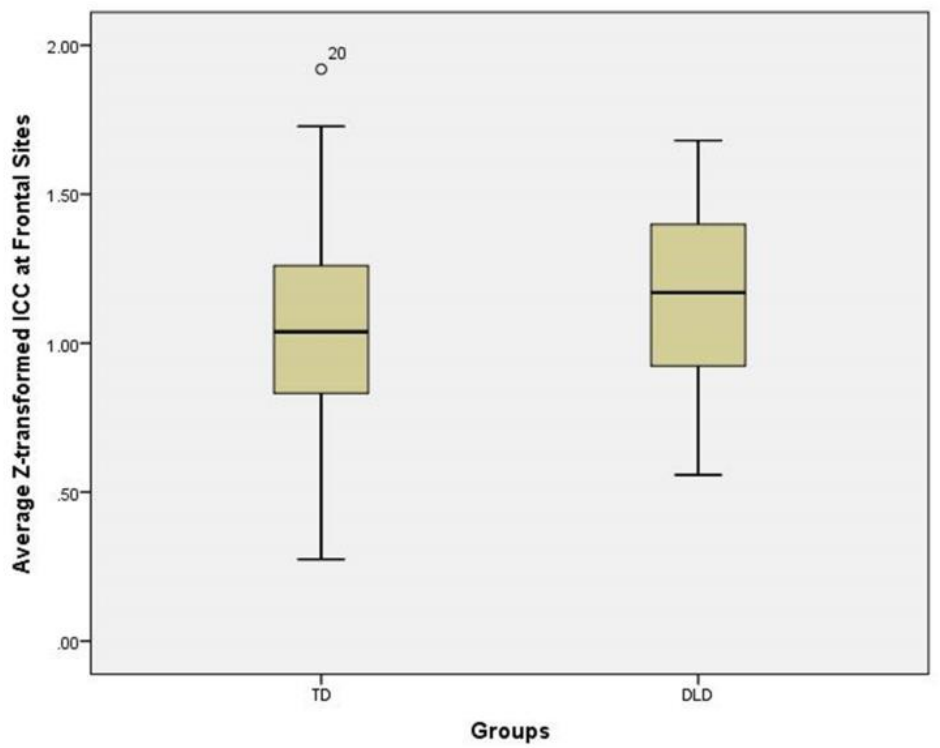

Figure 1. The distribution of average Fisher's z-transformed maximum ICC from the three frontal channels in children with typical development (TD) and DLD.

\section{Do the Groups with DLD and TD Differ in AEP-age Overall?}

Comparison of the groups with DLD and TD on basic demographic variables revealed that they did not differ in chronological age (see Table 1). As expected based on the use of the CELF-4 Core Language score for group inclusion, the participants with DLD had significantly weaker language abilities than controls, falling on average 1.5 SD below the test mean. Although both groups were required to have received a WASI PIQ score of at least 85 for inclusion, the group with DLD had significantly lower PIQ scores than the group with TD. It is worth noting, however, that the mean PIQ of the group with DLD fell well within the average range. Comparison of AEP-age across the two groups revealed that, at the overall group level, the 
auditory cortical responses to a tone in participants with DLD were not different from those of children with TD, $t(86)=-0.95, p=0.35$.

Table 1

Age, Language, Nonverbal IQ and Maximum ICC of Children with TD and Children with $D L D$

\begin{tabular}{|c|c|c|c|c|c|}
\hline \multirow[t]{2}{*}{ Subject data } & \multicolumn{2}{|c|}{$\begin{array}{c}\mathrm{TD} \\
(n=67)\end{array}$} & \multicolumn{2}{|c|}{$\begin{array}{c}\text { DLD } \\
(n=21)\end{array}$} & \multirow[t]{2}{*}{$t$} \\
\hline & Mean & $S D$ & Mean & $S D$ & \\
\hline Age & 9.0 & 1.0 & 9.1 & 1.2 & -.51 \\
\hline CLS & 104 & 10 & 78 & 7 & $13.63 *$ \\
\hline PIQ & 109 & 15 & 98 & 12 & $3.61 *$ \\
\hline Z-transformed & 1.04 & .31 & 1.15 & .33 & -1.55 \\
\hline \multicolumn{6}{|l|}{ Maximum ICC } \\
\hline AEP-age & 8.04 & .80 & 8.24 & .86 & .35 \\
\hline \multicolumn{6}{|c|}{ Note. CLS = core language scores from Clinical Evaluation of Language Fundamentals-4; } \\
\hline $\mathrm{PIQ}=$ performa & & $4 b b r$ & tellig & & \\
\hline
\end{tabular}

\section{Are Chronological Age and AEP-age Significant Predictors of Language Ability in DLD?}

Hierarchical regressions revealed that chronological age alone did not predict significant variance in the language ability of participants with $\operatorname{DLD}, F(1,19)=0.765, p=0.393$. The addition of AEP-age to the regression model resulted in a significant $R^{2}$ change, $F(2,18)=$ 4.583, $\left.p=0.025, \Delta R^{2}=0.30\right)$. AEP-age was found to be a significant predictor of language ability $(p=0.011)$, whereas chronological age approached but did not reach significance as a predictor of language ability $(p=0.054)$. No significant violation of collinearity among the 
variables was observed (Tolerance $\geq 0.847, \mathrm{VIF} \leq 1.181$ ). Table 2 summarizes the results of this regression analysis.

Table 2

Predicting Language Ability using Chronological Age and AEP-age

\begin{tabular}{lccccccc}
\hline Variables & $t$ & $p$ & Standardized $\beta$ & $F$ & $d f$ & $R^{2}$ & $\Delta R^{2}$ \\
\hline Language ability & & & & & & & \\
Step 1 & & & & 0.765 & 1,19 & 0.039 & 0.039 \\
Chronological age & 0.875 & 0.393 & 0.197 & & & & \\
Step 2 & & & & 4.583 & 2,18 & 0.337 & $0.299^{*}$ \\
Chronological age & 2.059 & 0.054 & 0.429 & & & & \\
AEP-age & 2.848 & 0.011 & 0.594 & & & & \\
\hline
\end{tabular}

Note: ${ }^{*} p<0.05$ (two-tail)

\section{Do Children with More Severe Language Impairments Have More Immature AEPs?}

To explore whether immature waveforms might only be evident, or be evident to a greater extent, in certain participants with DLD, we performed a subgroup analysis based on language ability. Participants with moderate-severe DLD had a significantly lower CELF-4 Core Language score than participants with mild DLD $(M=75.3$ and 82.7, respectively; $t(19)=-2.88$, $p=0.013)$. AEP-age of the participants with moderate-severe DLD $(M=7.92$ years, $S D=0.62)$ was compared to those with mild DLD $(M=8.67, S D=0.99)$. Participants with moderate-severe DLD had a significantly younger AEP-age than participants with mild DLD, $t(19)=3.515, p=$ 0.046, despite the fact that these two groups did not differ in chronological age $(M=9.21$ versus 8.98 years, respectively, $t(19)=1.45, p=0.656)$ or PIQ $(M=97.4$ and 98.8 , respectively, $t(19)=$ 
$-0.251, p=0.204)$. We also confirmed the similarity in chronological age between the two groups by comparing the proportion of children who were younger or older than the mean age of each group. Five of nine (56\%) participants in the mild DLD subgroup and seven of twelve (58\%) participants in the moderate-severe DLD subgroup were older than the mean age. A Chisquare test revealed no group difference in the distribution of age across the two subgroups $(p=$ 0.90). On average, the AEPs of participants with mild DLD were similar to (within 3 months of) their chronological age, whereas the AEPs of participants with moderate-severe DLD lagged, on average, 15 months behind their chronological age. To explore the difference between AEP-age and chronological age at an individual level, paired t-tests were conducted. Participants in the mild DLD group had an AEP-age similar to their own chronological age, $t(8)=0.457, p=0.66$, whereas participants with moderate-severe DLD had an AEP-age significantly younger than their chronological age $t(11)=3.515, p=0.005$.

These findings are consistent with visual inspection of the auditory cortical responses of the mild and moderate-severe DLD subgroups as plotted against the four normative age bands (see Figure 2). Participants with moderate-severe DLD had AEPs resembling typical controls who are 7 or 8 years old, while those with mild DLD had AEPs more similar to children with TD aged 9 to 10 years. The topological distribution of AEPs of participants with mild or moderatesevere DLD are shown in Figure 3. Overall, AEP amplitude (represented by color intensity) is larger for participants with moderate-severe DLD. The intensity and distribution of the topology in these children resembles typical controls who are 7 and 8 years old. In contrast, participants with mild DLD resemble the 9- to 10-year-olds with TD. One developmental change in topology seems particularly notable. Among younger children with TD, a marked bilateral negative response is observed at $199 \mathrm{~ms}$ post-stimulus at temporal electrodes. Such negativity becomes 
less pronounced at temporal sites and more dispersed towards prefrontal electrodes with age. Children with moderate-severe DLD showed a similar bilateral negativity pattern to younger children with TD, while children with mild DLD show topology resembling the prefrontal distribution of older children with TD.

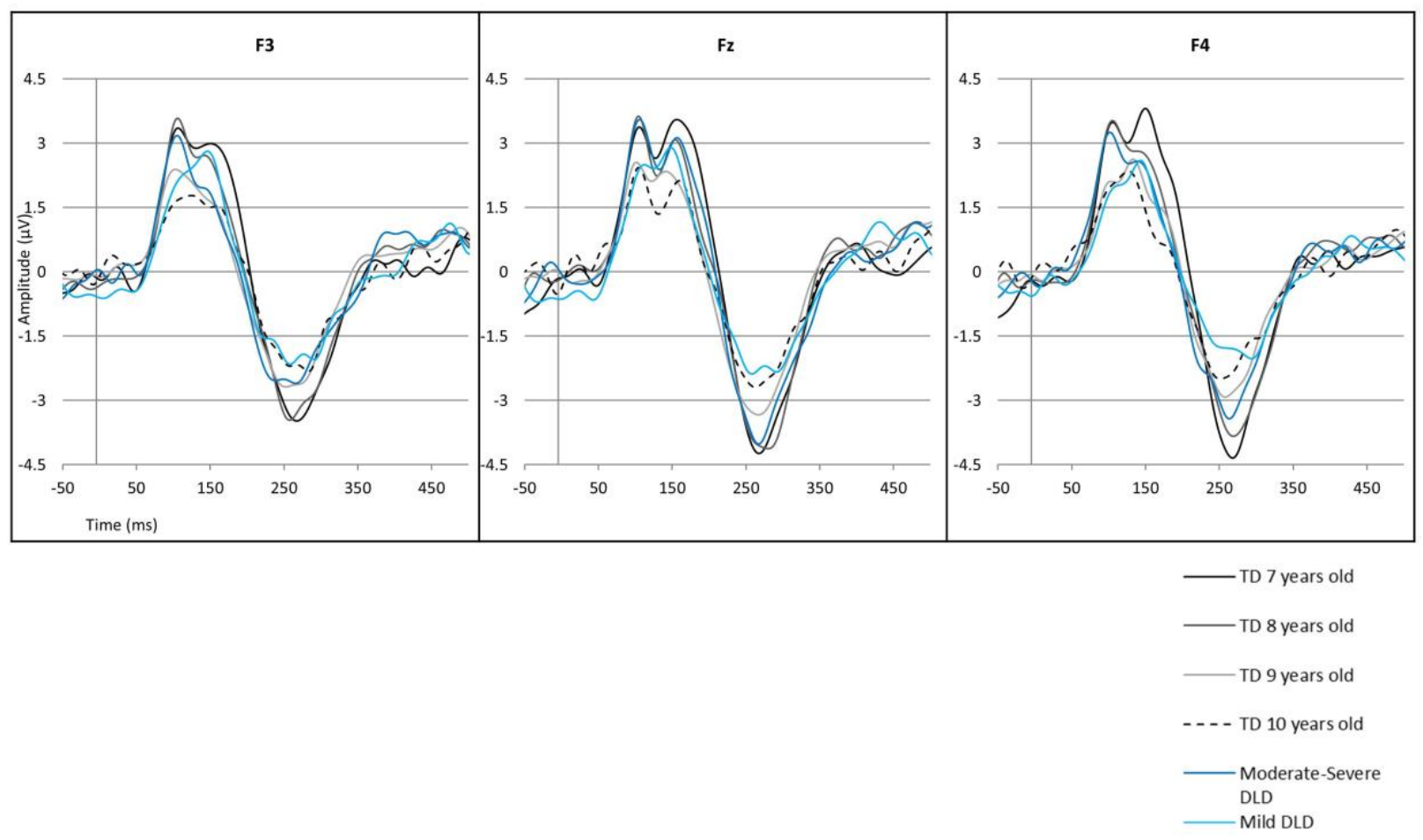

Figure 2. AEPs of children with DLD who had CELF-4 core language scores in the mild (between $10-15^{\text {th }}$ percentile) or moderate-to-severe (below $10^{\text {th }}$ percentile) ranges of impairment overlaid on four normative waveforms at three channels (from left to right: channels F3, Fz, F4). Amplitude in $\mu \mathrm{V}$ is plotted again post-stimulus presentation time in ms. 


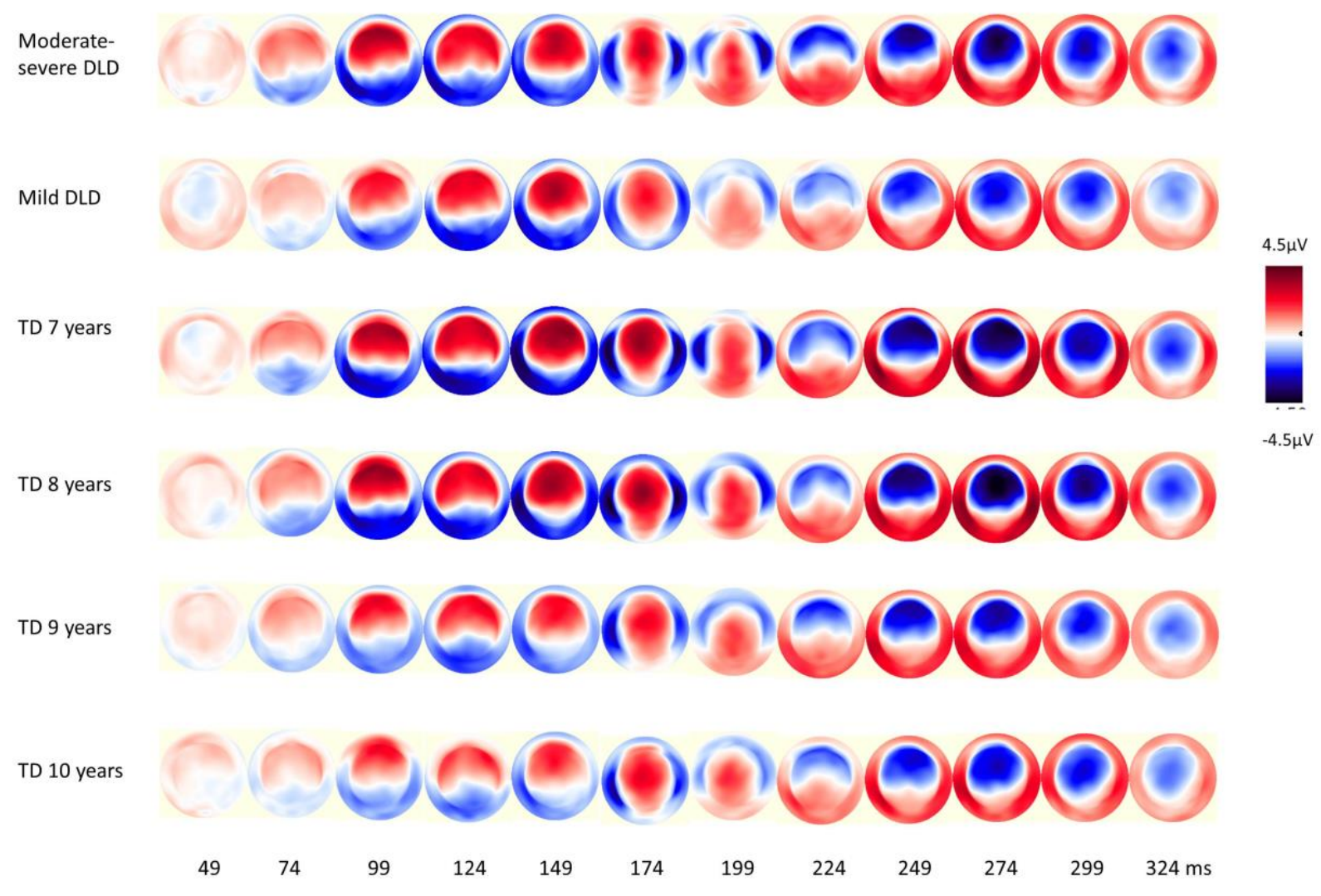

Figure 3. Topographic map of AEP amplitude between $49 \mathrm{~ms}$ to $324 \mathrm{~ms}$ post-stimulus presentation. Data from children with mild DLD and moderate-severe DLD as well as the four normative age groups derived from children with typical development are shown.

\section{Does AEP Maturation Predict Both Receptive and Expressive Language Abilities?}

Separate regression analyses were conducted to explore the contribution of AEP-age in receptive and expressive language abilities. Results revealed that AEP-age significantly predicted $20.6 \%$ of the variance in receptive language, $\beta=-0.454, F(1,19)=4.935, p=0.039$, but did not predict significant variance in expressive language $F(1,19)=2.043, p=0.169$. 


\section{Discussion}

\section{Overall Differences between DLD and TD in AEP maturity}

Our primary goal was to examine the immature auditory processing account of DLD by estimating AEP maturity in individual participants with DLD. We first ran an analysis on maximum ICCs and confirmed that our normative AEP waveforms from 7-10-year-old children with typical development could fully represent the maturity of AEP responses from participants with DLD. Then, we estimated AEP-age for each participant with DLD according to the normative waveform that most closely resembled that child's AEP. Contrary to our expectations, as a group, participants with DLD did not show a younger mean AEP-age compared to participants with typical development. Had we stopped our analyses at this point, this finding might have led us to conclude that our results failed to support the auditory immaturity hypothesis of DLD. However, as discussed in the introduction, significant inconsistencies have been found in the literature investigating AEP differences between children with DLD and TD. Alternative accounts have been put forward by Bishop et al. (2007) and McArthur and Bishop (2004b), who provided a critical review of inconsistent findings in the current literature and argued for the need to understand DLD at levels beyond group means.

\section{Predicting Language Ability from AEP Maturity and Considering Severity Subgroups}

Motivated by the above concerns, our pursuit of additional regression and subgroup analyses (research questions 2 and 3) provided further details about the nature of AEP maturation in children with DLD that may account for the lack overall group differences in our study and in several prior studies. Hierarchical regression analysis revealed that AEP-age explained $31 \%$ of the variance in language ability across our entire sample of children with DLD. The further stratification of our DLD sample into severity subgroups provided evidence of maturational 
delay in children with DLD who scored at or below the $10^{\text {th }}$ percentile on our language measure, that is, in those we identified as having moderate-severe DLD. Children with mild DLD appeared to have few delays, if any, in the maturation of their auditory cortical response. In sum, our whole group analysis might have led to the conclusion that DLD is not associated with immature auditory cortical processing because the relation, which in fact does appear to exist, is more nuanced (i.e., restricted to those with more significant degrees of language impairment). The inclusion of children with milder DLD in our initial whole group comparison likely limited the difference in AEP-age between children with DLD and TD.

Our regression analysis showed a much stronger relation between auditory processing of non-speech stimuli and standardized language performance in our DLD sample than in our prior sample of children with TD. In our study of children with TD, AEP-age was the dominant predictor of chronological age (accounting for $17 \%$ of variance), but explained a smaller (albeit significant) amount of variance (8\%) in language ability (Kwok et al., in press). The pattern of findings in the regression analyses across our two studies suggests that auditory cortical maturation may have a more prominent role in language development that is impaired than it does in typical language development. Our current subgroup analysis added an additional suggestion that this relation may be particularly prominent in, or restricted to, children with more substantive degrees of language impairment, although specific confirmation through regression analysis would require a larger sample of children with moderate-severe DLD. Assuming that our pattern of findings can be replicated, it raises a number of questions about why immature AEPs would only be found in children with moderate-severe DLD. 
One possible interpretation of differential auditory cortical maturation in children with different severities of language impairment is that this reflects different neural underpinnings in children with lesser versus greater degrees of impairment. Children who function within what we have termed the mild DLD range $\left(11-16^{\text {th }}\right.$ percentile) had auditory cortical responses that were appropriate for their age, raising the possibility that the language challenges of these children might may be related to factors other than auditory processing. Whether this points to different neural underpinning of language impairment in those with mild DLD, or suggests these children merely represent the low end of the normal distribution (i.e., that they may not have a disorder) is unclear, but worthy of further study using a large sample of children functioning in this range.

Our study does not allow for explicit conclusions about what neural underpinnings might be reflected by auditory cortical immaturity in children with moderate-severe DLD. Moreover, we are unable to address the important question of whether this immaturity is isolated to the auditory system, rather than part of a broader immaturity that extends beyond the auditory cortex. However, it is worth considering how our findings might relate to other neural correlates of DLD, both for speculating on how AEP immaturity might connect with previously reported structural and functional differences in the DLD population, and for highlighting how this immaturity might indeed be predominantly in the auditory system. As mentioned in the Introduction, P1 and N2 are particularly prominent in the AEPs of children in the developmental age range we studied here. The neural generators of P1 and N2 have been localized to the auditory cortex (Ponton et al., 2002; Eggermont \& Ponton, 2002), more specifically to Heschl's gyrus in the temporal lobe (Liegeois-Chauvel, Musolino, Badier, Maequis, \& Chauvel, 1994; Takeshita et al., 2002). Functionally, Heschl's gyrus and surrounding regions have been linked to phonological processing, including processing of speech-related cues for language learning 
(Hickok \& Poeppel, 2007; Warrier et al., 2009). In Magnetic Resonance Imaging studies, children with DLD have demonstrated a pattern of reduced hemispheric asymmetry in this perisylvian region relative to children with TD (see reviews by Leonard, 2014; Bishop, 2013), and this atypical anatomy may have functional consequences for auditory and spoken language processing (e.g., de Guibert et al., 2011; Hugdahl et al., 2004). In typical development, L>R functional asymmetry during auditory processing increases with age (Minagawa-Kawai, Cristià

\& Dupoux, 2011), raising the possibility that prior findings of atypical symmetry in children with DLD may reflect immature cortical development. In other words, increasing asymmetry may reflect movement to more mature, focal and lateralized processing and representation of speech and language, from more diffuse, symmetric processing in those with less developed language, be it younger, typical language learners or older children with DLD. Indeed, Bishop (2013) has proposed that diffuse, bilateral patterns of auditory processing seen in children with DLD may reflect a persistent pattern of immature cortical development. Our study provides neurophysiological support for this concept in that the patterns of children with DLD were like those of younger children with typical development, and further suggests that this concept may only apply to those with moderate-severe levels of impairment. Relating our findings to developing cerebral lateralization in the perisylvian region in children with and without DLD would be an important next step for future study.

\section{Impact of language score cut-offs}

One possible extension of our differential findings for our mild versus moderate-severe groups with DLD is that our neural data may be informative in the consideration of ideal test score cut-offs for language impairment classification in clinical and research samples. That is, the distinction between children with mild versus moderate-severe DLD in their cortical 
responses to sound could be taken as neurally based support for the use of the $10^{\text {th }}$ percentile (roughly 1.25 SD below the mean) rather than a more liberal cut-off of $1 \mathrm{SD}$ below the mean. However, determining who should be diagnosed with DLD is much more complex than determining what cut-off to use. Not only has the field moved beyond using an arbitrary standardized test score cut-off as the single, or even primary, determinant of which children should be diagnosed with DLD (see Leonard, 2014 for a review), Spaulding, Plante and Farinella (2006) have convincingly demonstrated that the cut-off providing the maximum sensitivity and specificity in identifying language impairment varies considerably across commonly used standardized tests of oral language. Nonetheless, many studies, including our own, continue to employ a particular standardized test score cut-off for inclusion/exclusion in DLD groups. What our results suggest is that for studies directed at identifying and understanding auditory cortical underpinnings of DLD, a $10^{\text {th }}$ percentile cut-off, at least on the $C E L F-4$, seems to matter.

Our results also demonstrate that the chosen cut-off for inclusion in a group of children with DLD can have a considerable impact on outcomes, in our case, in determining whether or not an auditory immaturity is found. This raises the possibility that language test cut-off may have been an important contributor to the contradictory findings in some of the prior psychobehavioural and neural investigations of the immature auditory processing account of DLD, and possibly in other research examining candidate cognitive, perceptual, or neural markers of DLD. Re-examination of these findings with the particular measure(s) and inclusion cut-offs used across studies in mind may shed light on this possible influence.

\section{Relation of AEP Maturity to Receptive vs Expressive Language Ability}

We also investigated the relative contribution of receptive and expressive language to AEP maturation. Receptive but not expressive language was found to significantly explain 
variance in AEP-age. This finding is consistent with the unique role of receptive language in AEP found by both Bishop et al. (2007) and Oram Cardy et al. (2008). This relation between AEP maturation and receptive language is currently not clear. It is possible that the maturity of sound perception, as reflected by AEP maturation, plays a direct role in the development of receptive language, whereas expressive language development is supported by other cognitive processes. In particular, the expressive language subtest, Recalling Sentences, has been shown to not only relate to semantic and synthetic knowledge but also to tap into working memory (Alloway, Gathercole, Willis, \& Adams, 2004). However, because our analysis was limited to single subtest measures of both receptive and expressive language, more detailed analysis is needed for future investigation of the specific relation between receptive and expressive language and AEP maturation.

\section{Limitations}

Certainly, an alternate explanation of our finding of auditory immaturity principally in children with moderate-severe DLD is that this was merely an artifact of sample size. It is possible that with a larger number of children in the mild DLD range, we might find significant, albeit small, degrees of immaturity in AEP-age for this group as well. Our differential findings across our two studies for the proportion of variance in auditory cortical maturation accounted for by language ability in children with DLD (31\%) versus those with typical development (8\%) suggests that the relation may not be linear across the childhood population as a whole. This pattern would not necessarily lead to a prediction that the degree of AEP-age immaturity relates in the same way to language ability across all ability levels, including in children in the mild DLD range. However, only a larger sample could provide confirmation. 
Also related to our limited sample size, we were unable to fully explore the role of performance IQ and auditory cortical maturity. Performing within normal limits on the performance IQ measure was one inclusion criterion for participants in the DLD group, hence, it is unlikely that our conclusions about auditory immaturity in the moderate-severe DLD group were simply a result of global intellectual impairment. However, the relative contribution of broader cognitive abilities versus language ability specifically in auditory cortical maturation should be explored in a larger sample of children with DLD.

Further investigation of the interaction between AEP-age and chronological age in predicting language ability is also warranted. While AEP-age was the only predictor that met our a priori cut-off for significance (i.e., $p<0.05)$, chronological age approached significance $(p=$ 0.054). The $p$-value between chronological age and language ability was also lower in step 2 compared to step 1 of the hierarchical regression analysis (Table 2). This suggests a possible interaction between the two predictors (i.e., chronological age and AEP-age) that impacted their relation with language ability, and that our sample was too small to detect such a relation. One possible interpretation of such an interaction effect would be that AEP-age is a stronger predictor of language ability for younger than older children. One way to investigate such interaction would be to compare the role of AEP-age in younger versus older children with DLD (i.e., by conducting separate regression analyses on younger versus older groups). This possible interaction effect would need to be explored further with a larger sample.

A final note of caution relates to our analytic method. Although the ICC method takes into account differences in the peak latency, amplitude, and morphology of AEP components, it does share some limitations with traditional peak analysis. Comparison of grand averages limits our ability to investigate the underlying sources of differences in grand averages that result in 
low ICC values. For example, a smaller AEP peak amplitude can be result from (a) a less pronounced electrophysiological response from the underlying neural generators, or (b) inconsistent timing of electrophysiological responses across trials resulting in a poor temporal alignment of peak amplitude during averaging. Other EEG analytic methodologies such as single-trial analysis may be able to reveal physiological contributions to immature AEP responses in future research.

\section{Conclusion}

Our results support the auditory immaturity hypothesis in the moderate-severe DLD subgroup. Certainly, caution should be exercised in generalizing this finding to different age ranges than the 7-10 year old range studied here. Due to the variability in developmental trajectories of the AEP, ICC may not be a sensitive measure for all ages. The reliability of the ICC method in the discrimination of children beyond the age range of the current study would require further investigation. Caution should be also exercised in attributing causality; the current findings provide little insight into the direction of the relation between auditory cortical maturation and language development. Assuming maturational delay in auditory processing is one of the cognitive-perceptual deficits that hinders language development, we should expect to find such delays in younger children with DLD. By contrast, if auditory cortical immaturity results from language impairment, then such delay may only be evident at later developmental stages. Information on longitudinal changes in auditory processes and auditory cortical maturation across various ages of DLD may provide more insights to the directionality of the relation between the two. To this end, we are exploring the sensitivity of ICC in discerning typical development of AEPs at various ages in our ongoing work. 
Certainly, much work needs to be completed before we may be able to draw direct clinical implications from this line of research. However, if future research supports the hypothesis that immaturities in the brain's ability to process basic acoustic signals is a biomarker for DLD, we might be able to one day use such a marker to predict which children are at risk for DLD when, or even before, they are late to talk.

\section{Acknowledgements}

We thank the children and parents for their participation, and Margot Stothers, Heather Brown, Rachael Smyth, Jillian Spratt, Jessica Boehm, Charity McCarthy, Jackson Wilson, Asha Shelton, and Katherine Harder for their assistance with data collection.

\section{References}

Alloway, T. P., Gathercole, S. E., Willis, C., \& Adams, A.-M. (2004). A structural analysis of working memory and related cognitive skills in young children. Journal of Experimental Child Psychology, 87(2), 85-106. http://doi.org/10.1016/j.jecp.2003.10.002

Archibald, L. M. D., \& Joanisse, M. F. (2012). Atypical neural responses to phonological detail in children with developmental language impairments. Developmental Cognitive Neuroscience, 2(1), 139-51. http://doi.org/10.1016/j.den.2011.07.003

Archibald, L. M. D., Oram Cardy, J., Joanisse, M. F., \& Ansari, D. (2013). Language, reading, and math learning profiles in an epidemiological sample of school age children. PloS One, 8(10), e77463. http://doi.org/10.1371/journal.pone.0077463

Bishop, D. V. M. (2007). Using mismatch negativity to study central auditory processing in developmental language and literacy impairments: where are we, and where should we be going? Psychological Bulletin, 133(4), 651-672. http://doi.org/10.1037/00332909.133.4.651

Bishop, D. V. (2013). Cerebral asymmetry and language development: cause, correlate, or consequence? Science, 340(6138), 1230531.

Bishop, D. V. M., Anderson, M., Reid, C., \& Fox, A. M. (2011). Auditory development between 7 and 11 years: an event-related potential (ERP) study. PloS One, 6(5), e18993. http://doi.org/10.1371/journal.pone.0018993

Bishop, D. V. M., \& Edmundson, A. (1987). Specific language impairment as a maturational lag: evidence from longitudinal data on language and motor development. Developmental Medicine \& Child Neurology, 29(4), 442-459. http://doi.org/10.1111/j.1469- 
8749.1987.tb02504.x

Bishop, D. V. M., Hardiman, M., Uwer, R., \& von Suchodoletz, W. (2007). Atypical longlatency auditory event-related potentials in a subset of children with specific language impairment. Developmental Science, 10(5), 576-87. http://doi.org/10.1111/j.14677687.2007.00620.x

Bishop, D. V. M., \& McArthur, G. M. (2004). Immature cortic

al responses to auditory stimuli in specific language impairment : evidence from ERPs to rapid tone sequences. Developmental Science, 4(7), 11-18. http://doi.org/10.1111/j.1467-

7687.2004.00356.x

Bishop, D. V. M., \& McArthur, G. M. (2005). Individual differences in auditory processing in specific language impairment: a follow-up study using event-related potentials and behavioural thresholds. Cortex, 41(3), 327-341. http://doi.org/10.1016/S00109452(08)70270-3 
Bishop, D. V. M., Snowling, M. J., Thompson, P. A., Greenhalgh, T., and The CATALISE-2 Consortium (2017). Phase 2 of CATALISE: a multinational and multidisciplinary Delphi consensus study of problems with language development: Terminology. Journal of Child Psychology and Psychiatry. http://doi.org/10.1111/jcpp.12721

Ceponiene, R., Cummings, A., Wulfeck, B., Ballantyne, A., \& Townsend, J. (2009). Spectral vs. temporal auditory processing in specific language impairment: a developmental ERP study. Brain and Language, 110(3), 107-20. http://doi.org/10.1016/j.bandl.2009.04.003

Ceponiene, R., Rinne, T., \& Näätänen, R. (2002). Maturation of cortical sound processing as indexed by event-related potentials. Clinical Neurophysiology, 113(6), 870-82. http://doi.org/10.1016/S1388-2457(02)00078-0

Choudhury, N., \& Benasich, A. a. (2011). Maturation of auditory evoked potentials from 6 to 48 months: Prediction to 3 and 4 year language and cognitive abilities. Clinical Neurophysiology, 122(2), 320-338. http://doi.org/10.1016/j.clinph.2010.05.035

Conti-Ramsden, G., Botting, N., \& Faragher, B. (2001). Psycholinguistic markers for specific language impairment (SLI). Journal of Child Psychology and Psychiatry, 42(6), 741-748. http://doi.org/10.1111/1469-7610.00770

Corriveau, K., Pasquini, E., \& Goswami, U. (2007). Basic auditory processing skills and specific language impairment: a new look at an old hypothesis. Journal of Speech, Language, and Hearing Research, 50(3), 647-66. http://doi.org/10.1044/1092-4388(2007/046)

de Guibert, C., Maumet, C., Jannin, P., Ferré, J. C., Tréguier, C., Barillot, C., ... \& Biraben, A. (2011). Abnormal functional lateralization and activity of language brain areas in typical specific language impairment (developmental dysphasia). Brain, 134, 3044-3058.

DeThorne, L. S., \& Schaefer, B. A. (2004). A guide to child nonverbal IQ measures. American Journal of Speech-Language Pathology, 13(4), 275. http://doi.org/10.1044/10580360(2004/029)

Duffy, F., Eksioglu, Y., \& Rotenberg, A. (2013). The frequency modulated auditory evoked response (FMAER), a technical advance for study of childhood language disorders: cortical source localization and. BMC, 13(1).

Eggermont, J. J., \& Ponton, C. W. (2002). The neurophysiology of auditory perception: from single units to evoked potentials. Audiology and Neurotology, 7, 71-99.

Eggermont, J. J., \& Ponton, C. W. (2003). Auditory-evoked potential studies of cortical maturation in normal hearing and implanted children: correlations with changes in structure and speech perception. Acta oto-laryngologica, 123(2), 249-252.

Hickok, G., \& Poeppel, D. (2007). The cortical organization of speech processing. Nature Reviews Neuroscience, 8, 393-402. 
Hugdahl, K., Gundersen, H., Brekke, C., Thomsen, T., Rimol, L. M., Ersland, L., \& Niemi, J. (2004). fMRI brain activation in a Finnish family with specific language impairment compared with a normal control group. Journal of Speech, Language, and Hearing Research, 47, 162-172.

Liegeois-Chauvel, C., Musolino, A., Badier, J. M., Marquis, P., \& Chauvel, P. (1994). Evoked potentials recorded from the auditory cortex in man: evaluation and topography of the middle latency components. Electroencephalography and Clinical Neurophysiology/Evoked Potentials Section, 92, 204-214.

Joanisse, M. F. (2004). Specific language impairments in children. Phonology, semantics, and the snglish past tense. Current Directions in Psychological Science, 13(4), 156-160. http://doi.org/10.1111/j.0963-7214.2004.00297.x

Kwok, E., Joanisse, M. F., Archibald, L. M. D., Stothers, M. E., Brown, H., \& Oram Cardy, J. (2018). Maturation in auditory event-related potentials predicts language ability in children. European Journal of Neuroscience, 47, 69-76.

Leonard, L. B. (2014). Children with specific language impairment (2nd ed.). Cambridge, MA: MIT Press.

Lincoln, A. J., Courchesne, E., Harms, L., \& Allen, M. (1995). Sensory modulation of auditory stimuli in children with autism and receptive developmental language disorder: eventrelated brain potential evidence. Journal of Autism and Developmental Disorders, 25(5), 521-539. http://doi.org/10.1007/BF02178298

McArthur, G. M., Atkinson, C., \& Ellis, D. (2009). Atypical brain responses to sounds in children with specific language and reading impairments. Developmental Science, 12(5), 768-783. http://doi.org/10.1111/j.1467-7687.2008.00804.x

McArthur, G. M., \& Bishop, D. V. M. (2004a). Frequency discrimination deficits in people with specific language impairment: reliability, validity, and linguistic correlates. Journal of Speech, Language, and Hearing Research, 47(3), 527-41. http://doi.org/10.1044/10924388(2004/041)

McArthur, G. M., \& Bishop, D. V. M. (2004b). Which people with specific language impairment have auditory processing deficits? Cognitive Neuropsychology, 21(1), 79-94. http://doi.org/10.1080/02643290342000087

McArthur, G. M., \& Bishop, D. V. M. (2005). Speech and non-speech processing in people with specific language impairment: a behavioural and electrophysiological study. Brain and Language, 94(3), 260-73. http://doi.org/10.1016/j.bandl.2005.01.002

Minagawa-Kawai, Y., Cristià, A., \& Dupoux, E. (2011). Cerebral lateralization and early speech acquisition: A developmental scenario. Developmental Cognitive Neuroscience, 1, 217-232.

Neville, H. J., Coffey, S. A., Holcomb, P. J., \& Tallal, P. (1993). The neurobiology of sensory and language processing in language-impaired children. Journal of Cognitive Neuroscience, 
5(2), 235-253. http://doi.org/10.1162/jocn.1993.5.2.235

Moore, J. K. (2002). Maturation of human auditory cortex: implications for speech perception. Annals of Otology, Rhinology \& Laryngology, 111(5_suppl), 7-10.

Oram Cardy, J., Flagg, E. J., Roberts, W., Brian, J., \& Roberts, T. P. L. (2005). Magnetoencephalography identifies rapid temporal processing deficit in autism and language impairment.

Oram Cardy, J., Flagg, E. J., Roberts, W., \& Roberts, T. P. L. (2008). Auditory evoked fields predict language ability and impairment in children. International Journal of Psychophysiology, 68(2), 170-5. http://doi.org/10.1016/j.ijpsycho.2007.10.015

Ors, M., Lindgren, M., Blennow, G., Nettelbladt, U., Sahlén, B., \& Rosén, I. (2002). Auditory event-related brain potentials in children with specific language impairment. European Journal of Paediatric Neurology, 6(1), 47-62. https://doi.org/10.1053/ejpn.2001.0541

Ponton, C. W., Eggermont, J. J., Kwong, B., \& Don, M. (2000). Maturation of human central auditory system activity: Evidence from multi-channel evoked potentials. Clinical Neurophysiologyiology, 111(2), 220-36. http://doi.org/doi:10.1016/S1388-2457(99)002369

Reilly, S., Tomblin, B., Law, J., McKean, C., Mensah, F. K., Morgan, A., ... \& Wake, M. (2014). Specific language impairment: a convenient label for whom? International Journal of Language \& Communication Disorders, 49(4), 416-451.

Rice, M. L., Wexler, K., \& Hershberger, S. (1998). Tense over time: The longitudinal course of tense acquisition in children with specific language impairment. Journal of Speech Language and Hearing Research, 41(6), 1412. http://doi.org/10.1044/jslhr.4106.1412

Rubin, H., Kantor, M., \& Macnab, J. (1990). Grammatical awareness in the spoken and written language of language-disabled children. Canadian Journal of Psychology, 44(4), 483-500. http://doi.org/10.1037/h0084269

Semel, E., Wiig, E. H., \& Secord, W. A. (2003). Clinical Evaluation of Language Fundamentals (4th ed.). Toronto, ON: Psychological Corp.

Smith-Lock, K. M. (1993). Morphological analysis and the acquisition of morphology and syntax in specifically-language-impaired children. Haskins Laboratories Status Report on Speech Research, 113-138.

Spaulding, T. J., Plante, E., \& Farinella, K. A. (2006). Eligibility criteria for language impairment. Language Speech and Hearing Services in Schools, 37(1), 61. http://doi.org/10.1044/0161-1461(2006/007)

Sussman, E., Steinschneider, M., Gumenyuk, V., Grushko, J., \& Lawson, K. (2008). The maturation of human evoked brain potentials to sounds presented at different stimulus rates. Hearing Research, 236(1-2), 61-79. http://doi.org/10.1016/j.heares.2007.12.001 
Takeshita, K., Nagamine, T., Thuy, D. H. D., Satow, T., Matsuhashi, M., Yamamoto, J., ... \& Shibasaki, H. (2002). Maturational change of parallel auditory processing in school-aged children revealed by simultaneous recording of magnetic and electric cortical responses. Clinical Neurophysiology, 113, 1470-1484.

Tallal, P. (2000). Experimental studies of language learning impairments: From research to remediation. In D. V. M. Bishop \& L. B. Leonard (Eds.), Speech and Language Impairments in Children: Causes, Characteristics, Intervention and Outcome (pp. 131155). New York, NY: Psychology Press.

Tonnquist-Uhlén, I. (1996). Topography of auditory evoked long-latency potentials in children with severe language impairment: the P2 and N2 components. Ear \& Hearing, 17(4), 314326.

Uwer, R., Albrecht, R., \& von Suchodoletz, W. (2002). Automatic processing of tones and speech stimuli in children with specific language impairment. Developmental Medicine and Child Neurology, 44(8), 527-32.

Warrier, C., Wong, P., Penhune, V., Zatorre, R., Parrish, T., Abrams, D., \& Kraus, N. (2009). Relating structure to function: Heschl's Gyrus and acoustic processing. Journal of Neuroscience, 29, 61-69.

Wechler, D. (1999). Wechsler Abbreciated Scale of Intelligence. Toronto, ON: Psychological Corp.

Wible, B., Nicol, T., \& Kraus, N. (2005). Correlation between brainstem and cortical auditory processes in normal and language-impaired children. Brain, 128, 417-423.

Wright, B. A., \& Reid, M. D. (2002). Excessive auditory masking in children with language or listening impairments interpreted as developmental delay. Proceedings of the 25th Annual Meeting of the Association for Research in Otolaryngology, 25, 19-20. 